\title{
Biodiesel from low cost palm stearin using metal doped methoxide solid catalyst
}

\begin{abstract}
Heterogeneous transesterification of vegetable oils offers an environmentally more attractive option for biodiesel production compared to the conventional homogeneous processes. Thus, the metal doped methoxide catalyst was developed in the present study which aims to improve the transesterification of low cost palm stearin (PS) and reduce the generation of waste. The catalyst was characterized by X-ray diffraction (XRD), field emission scanning electron microscopy (FESEM), nitrogen adsorption/desorption (BET) and temperature programmed desorption-CO2 (TPD-CO2) coupled with mass spectrometer. The optimum parameters were studied via response surface methodology (RSM) coupled with central composite design (CCD). The synthesized catalyst exhibited a high biodiesel yield at $94.7 \%$ with methanol to PS molar ratio of $6: 1,1 \%$ catalyst loading and less than $3 \mathrm{~h}$ of reaction time. The better catalytic activity of the aforementioned catalyst in the biodiesel reaction could be attributed to the presence of optimal number of catalytically active acid site density on its surface.
\end{abstract}

Keyword: Biodiesel; Catalyst; Metal doped methoxide; Optimization; TPD-CO2 